\title{
On the spectral problem for trivariate functions
}

\author{
Behnam Hashemi · Yuji Nakatsukasa
}

Received: date / Accepted: date

\begin{abstract}
Using a variational approach applied to generalized Rayleigh functionals, we extend the concepts of singular values and singular functions to trivariate functions defined on a rectangular parallelepiped. We also consider eigenvalues and eigenfunctions for trivariate functions whose domain is a cube. For a general finite-rank trivariate function, we describe an algorithm for computing the canonical polyadic (CP) decomposition, provided that the $\mathrm{CP}$ factors are linearly independent in two variables. All these notions are computed using Chebfun3; a part of Chebfun for numerical computing with 3D functions. Application in finding the best rank-1 approximation of trivariate functions is investigated. We also prove that if the function is analytic and two-way orthogonally decomposable (odeco), then the CP values decay geometrically, and optimal finite-rank approximants converge at the same rate.
\end{abstract}

Keywords Trivariate functions - Singular values · Higher order power method $(\mathrm{HOPM}) \cdot$ Low-rank approximation $\cdot$ CP decomposition

Mathematics Subject Classification (2000) $65 \mathrm{~K} 10 \cdot 15 \mathrm{~A} 18 \cdot 15 \mathrm{~A} 69$

\section{Introduction}

The singular value decomposition (SVD) of a bivariate function was first introduced by Schmidt [47]. He proved existence, uniqueness and the best low rank approximation property of the SVD. Hammerstein and Lipschitz proved theorems regarding

B. Hashemi

Department of Mathematics, Shiraz University of Technology, Modarres BLVD., 71555-313 Shiraz, Iran, \& School of Mathematics, Institute for Research in Fundamental Sciences (IPM), P.O. Box: 19395-5746, Tehran, Iran.

E-mail: hashemi@sutech.ac.ir

Y. Nakatsukasa

National Institute of Informatics, 2-1-2 Hitotsubashi, Chiyoda-ku, Tokyo 101-8430, Japan

E-mail: nakatsukasa@nii.ac.jp 
the uniform and absolute convergence of the singular functions. The name "singular value" actually comes from integral equations, i.e., in connection to the continuous SVD. For more on the history see [51] and [57]. Bounds on the decay rate of singular values of analytic bivariate functions are given in [30, p. 313] and [57]. Hille and Tamarkin [25] summarized different decay rates for eigenvalues of bivariate functions. See also $[37,38,55]$ and $[50$, p. 379].

This paper considers the spectral problem for trivariate functions, by examining their singular values and eigenvalues. For the discrete case, a huge amount of work has been presented in the literature to discuss the spectral problems for tensors. Beginning from some of their continuous counterparts, we present the notions of singular values and eigenvalues of trivariate functions, discuss the connection to the canonical polyadic $(\mathrm{CP})$ decomposition and prove decay rates for the $\mathrm{CP}$ values of certain trivariate functions. We also present numerical algorithms for computing the aforementioned notions. One can also consider these concepts in a more abstract setting of tensor product Hilbert spaces [23], although this paper focuses on tensor products of $L^{2}$ functions.

The spectral theory of discrete tensors was initiated by Lim [36] and Qi [42] independently. We start by extending Lim's ideas to develop a spectral framework for trivariate functions. Our work involves bounded trilinear forms $\phi: L^{2}([a, b]) \times$ $L^{2}([c, d]) \times L^{2}([e, l]) \rightarrow \mathbb{R}$. Assuming $g(x) \in L^{2}([a, b]), h(y) \in L^{2}([c, d])$, and $w(z) \in$ $L^{2}([e, l])$, the action of $\phi$ on $g, h$ and $w$ is denoted by $\phi(g, h, w)$. Since Lim's derivation is based on a variational approach, we first present the variational derivative for trivariate functions.

Definition 1.1 The functional (variational) derivative of $\phi$ is

$$
\partial \phi:=\left[\begin{array}{c}
\partial_{g} \phi \\
\partial_{h} \phi \\
\partial_{w} \phi
\end{array}\right]
$$

where $\partial_{g} \phi, \partial_{h} \phi$ and $\partial_{w} \phi$ denote the partial functional derivatives of $\phi$ with respect to $g, h$ and $w$, respectively [22, p. 49]. Here, e.g., $\partial_{g} \phi$ is a function of $x, y$ and $z$ that satisfies

$$
\phi(g+\delta g, h, w)-\phi(g, h, w)=\int_{a}^{b} \partial_{g} \phi(x, y, z) \delta g(x) d x+\mathscr{O}\left(\delta g^{2}\right) .
$$

Analogous formulas hold for $\partial_{h} \phi$ and $\partial_{w} \phi$. See e.g., [1, p. 224], [9], [18, p. 405] and [49].

Note that the kernel of the integral on the right-hand side defines the partial variational derivative of the functional.

Given $f:[a, b] \times[c, d] \times[e, l] \rightarrow \mathbb{R}$, the problem of finding the best rank-1 approximation of $f$ is to compute the functions $g(x), h(y)$, and $w(z)$ which have unit 2-norms and a scalar $\sigma$ that solve

$$
\min \int_{a}^{b} \int_{c}^{d} \int_{e}^{l}(f(x, y, z)-\sigma g(x) h(y) w(z))^{2} d z d y d x
$$

This is equivalent to minimizing the $L^{2}$-norm of the residual $f(x, y, z)-\sigma g(x) h(y) w(z)$; a minimizer is guaranteed to exist [19, Thm. 4.1]. 
Throughout the paper $\|\cdot\|$ is the spectral norm of discrete tensors and multivariate functions, i.e.,

$$
\|f\|:=\sup \frac{\int_{a}^{b} \int_{c}^{d} \int_{e}^{l} f(x, y, z) g(x) h(y) w(z) d z d y d x}{\|g\|_{2}\|h\|_{2}\|w\|_{2}},
$$

where $\|g\|_{2}^{2}:=\int_{a}^{b}|g(x)|^{2} d x,\|h\|_{2}^{2}:=\int_{c}^{d}|h(y)|^{2} d y$, and $\|w\|_{2}^{2}:=\int_{e}^{l}|w(z)|^{2} d z$ are the $L^{2}$-norms of functions. As in the $2 \mathrm{D}$ case, the spectral norm turns out to be equal to the largest singular value, and its corresponding singular factors $g, h, w$ give the best rank-1 approximation (1.1). See Section 2.

One of the extensions of the SVD to higher-order tensors is the canonical polyadic (CP) decomposition, which is a decomposition of a discrete tensor into a sum of rankone tensors. The natural continuous analogue for trivariate functions is

$$
f(x, y, z)=\sum_{i=1}^{\infty} \alpha_{i} g_{i}(x) h_{i}(y) w_{i}(z)
$$

which is understood to hold in $L^{2}$, that is, almost everywhere. If there exists a finite minimal number $r$ of terms in (1.2), it is called the rank, in which case (1.2) is called the canonical polyadic $(\mathrm{CP})$ decomposition or expansion of $f$. Note that the concept of tensor rank is in general ill-posed without additional assumptions [15]. Even if $f$ does not have a finite rank, in practice one only keeps a finite number of terms to approximate $f$. In this work we derive a practical numerical algorithm for computing an approximate finite-rank CP decomposition of trivariate functions. Knowing a $\mathrm{CP}$ decomposition of $f(x, y, z)$ offers a number of advantages, including compressed representation and ease of manipulation, such as integration and differentiation.

As mentioned above, it is known that for bivariate functions the singular values decay geometrically if the function is analytic. We prove that for trivariate functions that are analytic and two-way odeco (which is less restrictive than (three-way) odeco), the CP-values $\alpha_{i}$ of admit a similar rate of decay, and best low-rank approximations can be obtained by truncating the $\mathrm{CP}$ decomposition.

Recently, spectral properties associated with other tensor formats have been studied $[7,21,43]$. For example, convergence theory of the functional tensor-train (FTT) approximation of an $n$-dimensional function $f$ relies on its singular values (cf. [7, Prop. 9]). Here, the singular values are defined as the square root of the eigenvalues of the operators $T T^{*}$ and $T^{*} T$ where $f$ is regarded as a Hilbert-Schmidt kernel of a certain integral operator $T$, and $T^{*}$ is the corresponding Hilbert adjoint operator.

This paper is organized as follows. In Section 2 we present the singular values and singular functions of trivariate functions. Section 3 deals with eigenvalues and eigenfunctions for trivariate functions of cube domain. In Section 4 we discuss the computation of the $\mathrm{CP}$ decomposition. Section 5 treats the decay of $\mathrm{CP}$ values for analytic trivariate functions that are (two-way) odeco, and their low-rank approximation. Section 6 presents numerical experiments to illustrate the notions and results.

\section{The singular value problem for trivariate functions}

Here we consider the singular value problem for trivariate functions. We first review the counterpart for discrete order-three tensors, and then present the singular value problem for trivariate functions as a natural extension. 
The singular vectors of a given discrete tensor $A \in \mathbb{R}^{m \times n \times p}$ are the stationary points of the following functional [36], [20, p. 739]:

$$
\left\{\begin{array}{l}
r_{A}: \mathbb{R}^{m} \times \mathbb{R}^{n} \times \mathbb{R}^{p} \rightarrow \mathbb{R}, \\
r_{A}(u, v, w):=\frac{\sum_{i=1}^{m} \sum_{j=1}^{n} \sum_{k=1}^{p} A(i, j, k) u(i) v(j) w(k)}{\|u\|_{2}\|v\|_{2}\|w\|_{2}} .
\end{array}\right.
$$

If $u, v$, and $w$ are unit vectors, then from the gradient of $r_{A}(u, v, w)$ we get the nonlinear system of equations

$$
\nabla r_{A}=\left[\begin{array}{c}
A_{(1)}(w \otimes v) \\
A_{(2)}(w \otimes u) \\
A_{(3)}(v \otimes u)
\end{array}\right]-r_{A}(u, v, w)\left[\begin{array}{c}
u \\
v \\
w
\end{array}\right]=0 .
$$

Here $A_{(i)}$ is the mode- $i$ unfolding [28, Sec. 2.4] of $A$ and $\otimes$ is the Kronecker product. The above equation can be solved by an iterative method like the power iteration. Applications include finding the nearest rank-1 tensor to $A$.

Next, let $f(x, y, z)$ be a trivariate function defined on the rectangular parallelepiped $[a, b] \times[c, d] \times[e, l]$. Analogous to (2.1), we define the singular values and singular functions of $f$ as the stationary points of the generalized Rayleigh functional

$$
\left\{\begin{array}{l}
r_{f}: L^{2}([a, b]) \times L^{2}([c, d]) \times L^{2}([e, l]) \rightarrow \mathbb{R} \\
r_{f}(g, h, w):=\frac{\int_{a}^{b} \int_{c}^{d} \int_{e}^{l} f(x, y, z) g(x) h(y) w(z) d z d y d x}{\|g\|_{2}\|h\|_{2}\|w\|_{2}} .
\end{array}\right.
$$

As before, we consider

$$
\max \int_{a}^{b} \int_{c}^{d} \int_{e}^{l} f(x, y, z) g(x) h(y) w(z) d z d y d x,
$$

subject to the constraint that

$$
\|g\|_{2}^{2}=\|h\|_{2}^{2}=\|w\|_{2}^{2}=1 .
$$

The Lagrangian $L_{f}(g, h, w)$ for the constraint optimization problem (2.4) is

$$
\begin{aligned}
& \int_{a}^{b} \int_{c}^{d} \int_{e}^{l} f(x, y, z) g(x) h(y) w(z) d z d y d x \\
& -\frac{\mu_{1}}{2} \int_{a}^{b}\left(g(x)^{2}-1\right) d x-\frac{\mu_{2}}{2} \int_{c}^{d}\left(h(y)^{2}-1\right) d y-\frac{\mu_{3}}{2} \int_{e}^{l}\left(w(z)^{2}-1\right) d z .
\end{aligned}
$$

Since

$$
\begin{aligned}
L_{f}(g+\delta g, h, w)-L_{f}(g, h, w) & =\int_{a}^{b}\left(\int_{c}^{d} \int_{e}^{l} f(x, y, z) w(z) h(y) d z d y\right) \delta g(x) d x \\
& -\mu_{1} \int_{a}^{b} g(x) \delta g(x) d x+O\left(\|\delta g\|^{2}\right),
\end{aligned}
$$

the partial functional derivative of $L_{f}(g, h, w)$ with respect to $g$ is

$$
\int_{c}^{d} \int_{e}^{l} f(x, y, z) w(z) h(y) d z d y-\mu_{1} g(x) .
$$


By computing the partial functional derivatives of $L_{f}$ with respect to $h$ and $w$ and setting them to zero, we obtain the following system at a critical point:

$$
\left\{\begin{array}{l}
\int_{c}^{d} \int_{e}^{l} f(x, y, z) w(z) h(y) d z d y=\mu_{1} g(x), \\
\int_{a}^{b} \int_{e}^{l} f(x, y, z) w(z) g(x) d z d x=\mu_{2} h(y), \\
\int_{a}^{b} \int_{c}^{d} f(x, y, z) h(y) g(x) d y d x=\mu_{3} w(z),
\end{array}\right.
$$

which together with the three equality constraints (2.5) form the KKT system. We now apply $g(x), h(y)$, and $w(z)$ to the first, second and last equations above, respectively to get

$$
\mu_{1}=\mu_{2}=\mu_{3}=\int_{a}^{b} \int_{c}^{d} \int_{e}^{l} f(x, y, z) g(x) h(y) w(z) d z d y d x=r_{f}(g, h, w) .
$$

Setting $\sigma:=r_{f}(g, h, w)$ we have

$$
\left\{\begin{array}{c}
\int_{c}^{d} \int_{e}^{l} f(x, y, z) w(z) h(y) d z d y=\sigma g(x), \\
\int_{a}^{b} \int_{e}^{l} f(x, y, z) w(z) g(x) d z d x=\sigma h(y), \\
\int_{a}^{b} \int_{c}^{d} f(x, y, z) h(y) g(x) d y d x=\sigma w(z), \\
\int_{a}^{b} \int_{c}^{d} \int_{e}^{l} f(x, y, z) g(x) h(y) w(z) d z d y d x=\sigma .
\end{array}\right.
$$

We call $(\sigma, g, h, w)$ satisfying (2.5) and (2.7) a singular quadruplet of $f$. Note from (2.7) that $(\sigma, g, h, w)$ is a singular quadruplet of $f$ if and only if the same is true for $(-\sigma,-g,-h,-w)$. We do not distinguish these and assume $\sigma \geq 0$ unless otherwise mentioned.

The singular quadruplet $\left(\sigma_{1}, g_{1}, h_{1}, w_{1}\right)$ with the largest $\sigma_{1}$ is called the largest singular quadruplet. Below is a natural continuous analogue of the well-known best rank-1 approximation property of the largest singular quadruplets for discrete tensors.

Theorem 2.1 The largest singular quadruplet $\left(\sigma_{1}, g_{1}, h_{1}, w_{1}\right)$ is the best rank-1 approximation to $f$, i.e., it is the solution for (1.1).

Proof As shown in [63] for the discrete case, we can verify that when $g, h$, and $w$ are fixed, the objective function in (1.1) under the constraints (2.5) is minimized when $\sigma=r_{f}(g, h, w)$, with the minimum value

$$
\min \int_{a}^{b} \int_{c}^{d} \int_{e}^{l}(f(x, y, z))^{2} d z d y d x-\left(r_{f}(g, h, w)\right)^{2} .
$$

Since this is clearly minimized when $\left|r_{f}(g, h, w)\right|$ is maximized, it follows that (1.1) is equivalent to the dual problem of maximizing the generalized Rayleigh functional (2.4).

2.1 Algorithms for computing a singular value

The higher order power method (HOPM) for discrete tensors is a generalization of the power method for discrete matrices [11,63]. Each iteration of the HOPM for an order three discrete tensor has three steps. At each step one of the three singular vectors is updated while the remaining two are kept unchanged and this process continues alternatively in the next two steps of each iteration. It is shown in [44] that the sequence 
of values of the functional (2.1) generated in this way is monotonic and therefore the Rayleigh functional converges ${ }^{1}$. See also [61] for further references and a recent convergence analysis of the singular factors.

Algorithm 1 is a continuous extension of the HOPM based on (2.7) that can be used to compute a singular quadruplet of $f(x, y, z)$. Essentially it is obtained by replacing tensor-vector products with integration. As in the discrete case [61], it can be viewed as an alternating least-squares algorithm.

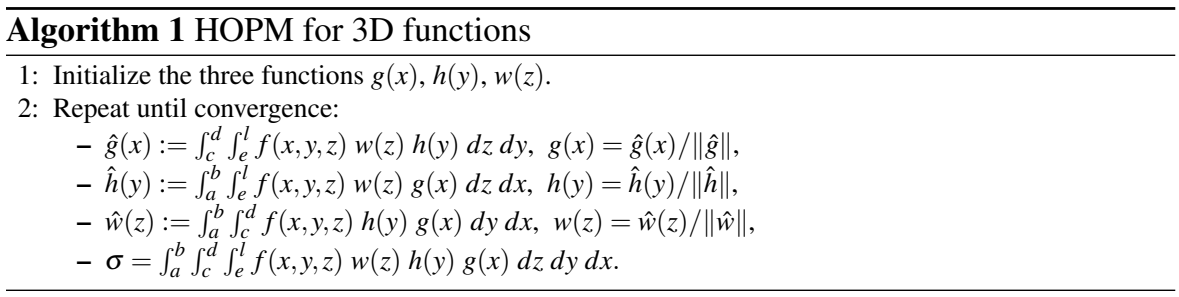

Algorithm 1 can be equivalently understood as follows: each step finds the minimizer of the $L^{2}$ norm $\|f-g h w\|_{L^{2}}$ with respect to one function (e.g. $g$ ), fixing the rest $(h, w)$. The minimization in turn is equivalent to the dual problem of maximizing the Rayleigh functional (2.3).

The monotonicity of the $\sigma$ values - which holds for discrete tensors as mentioned above-carries over to HOPM for (trivariate) functions as follows: for fixed $h$ and $g$, consider the functional $\sigma(w)=\int_{a}^{b} \int_{c}^{d} \int_{e}^{l} f(x, y, z) w(z) h(y) g(x) d z d y d x$. By the Cauchy-Schwarz inequality, $\sigma(w)$ is maximized over $\|w\|=1$ by taking $w$ to be a positive scalar multiple of $\int_{a}^{b} \int_{c}^{d} f(x, y, z) h(y) g(x) d y d x$, which is precisely the $w$ obtained by the update in HOPM. Therefore each HOPM update results in a monotonic nondecrease of $\sigma$. Since $\sigma$ is clearly bounded by $\|f\|$, it follows that the iterates $\sigma$ converge.

It is easy to implement Algorithm 1 in Chebfun. A chebfun3 object [24] constructed from a 3D function $f$ has the Tucker representation

$$
f(x, y, z) \approx T \times{ }_{1} A(x) \times{ }_{2} B(y) \times{ }_{3} C(z),
$$

where $T$ is a discrete tensor of size $r_{x} \times r_{y} \times r_{z}, A(x)$ is a quasimatrix of size $\infty \times$ $r_{x}$ defined over $[a, b], B(y)$ is a quasimatrix of size $\infty \times r_{y}$ over $[c, d]$ and $C(z)$ is a quasimatrix of size $\infty \times r_{z}$ defined over $[e, g]$. The above formula involves modal contractions of the core tensor $T$ and the quasimatrices $A(x), B(y)$ and $C(z)$ and can be rewritten as

$$
f(x, y, z) \approx \sum_{i=1}^{r_{x}} \sum_{j=1}^{r_{y}} \sum_{k=1}^{r_{z}} t_{i, j, k} a_{i}(x) b_{j}(y) c_{k}(z) .
$$

Either $\left(r_{x}, r_{y}, r_{z}\right)$ or $r:=\max \left\{r_{x}, r_{y}, r_{z}\right\}$ is called the numerical Tucker $\operatorname{rank}^{2}$ of $f$. Assuming that the number of Chebyshev coefficients needed to represent each univariate function $a_{i}(x), b_{i}(y)$, and $c_{i}(z)$ is $m, n$ and $p$, respectively, either $(m, n, p)$

\footnotetext{
1 Unless in the exceptional case that the initial vectors correspond to a saddle point of (2.1) [26, Thm. 2]

2 relative to a tolerance to which (2.10) holds; machine epsilon by default
} 
or $\max \{m, n, p\}$ is called the length of a chebfun3 object. Assuming for notational simplicity that $n=\max \{m, n, p\}$, the cost of constructing a chebfun3 object from $f(x, y, z)$ is $\mathscr{O}\left(n r^{4}+r n \log n\right)[24]$.

We claim that the cost of each iteration of Algorithm 1 is $\mathscr{O}\left(r^{3}+r n \log n\right)$. This can be verified as follows. The dominant cost of each iteration is the computation of the three double integrals. For example the first one can be computed as follows.

$$
\hat{g}(x)=T \times{ }_{1} A(x) \times{ }_{2} \underbrace{\left(\int_{c}^{d} B(y) h(y) d y\right)}_{\text {an } r \times 1 \text { vector }} \times \underbrace{\left(\int_{e}^{l} C(z) w(z) d z\right)}_{\text {another } r \times 1 \text { vector }}
$$

Each column of $B(y)$ has length $n$ and the inner product of two 1D chebfuns using Clenshaw-Curtis quadrature needs $\mathscr{O}(n \log n)$ operations [59, Chap. 19] (the initial functions in step 1 are assumed to have lengths bounded by $n$; then the degrees are bounded by $n-1$ throughout the iterations). Since $B(y)$ has $r$ columns, applying $B(y)$ to $h(y) \operatorname{costs} \mathscr{O}(r n \log n)$. Similarly, the second integral above needs $\mathscr{O}(r n \log n)$ arithmetic operations. The modal multiplication of the $r \times r \times r$ core tensor $T$ with an $r \times 1$ discrete vector needs $\mathscr{O}\left(r^{3}\right)$ operations and produces a matrix of size $r \times r$. We then multiply the matrix with the vector resulting from the second integral above. Finally the resulting vector is used to form a linear combination of the columns of $A(x)$ to compute $\hat{g}(x)$. Hence the overall cost is $\mathscr{O}\left(r^{3}+r n \log n\right)$.

Therefore, the cost of forming a chebfun3 object plus that of using Algorithm 1 in Chebfun is quartic in $r$ and only log-linear in the length of the chebfun 3 object. It is therefore clear that Algorithm 1 is particularly efficient for chebfun3 object that are already constructed from low rank functions. Note that the cost of each iteration of HOPM for discrete full tensors of size $n \times n \times n$ is $\mathscr{O}\left(n^{3}\right)$ since this is the cost of tensor-vector contractions. The cost reduces to $\mathscr{O}\left(r^{3}+r n\right)$ provided that the Tucker decomposition of the discrete tensor is already available; this is comparable to the cost of working with a chebfun 3 object.

If we apply the continuous analogue of the higher order SVD [12] to the above Tucker representation of $f$, then we get

$$
f(x, y, z) \approx \hat{f}(x, y, z)=\hat{T} \times{ }_{1} \hat{A}(x) \times{ }_{2} \hat{B}(y) \times{ }_{3} \hat{C}(z),
$$

in which the columns of the factor quasimatrices $\hat{A}(x), \hat{B}(y)$ and $\hat{C}(z)$ are orthonormal and the discrete core tensor $\hat{T}$ is all orthogonal, i.e., its horizontal, lateral and frontal slices are all orthogonal matrices. An alternative to Algorithm 1 for computing a singular quadruplet of a chebfun3 object is as follows. Using Chebfun's command hosvd we compute the higher-order SVD of the chebfun3 object where the factor quasimatrices have unit 2-norm. Then we simply apply the discrete version of HOPM, based on (2.2), to the core tensor $\hat{T}$ of the HOSVD of $f$ in (2.11). See Algorithm 2.

In Step 2 of Algorithm 2, the discrete HOPM can be replaced by any other algorithm that computes the singular values and singular vectors of a discrete tensor. Assuming that we first construct a chebfun3 object from the input function handle in both Algorithm 1 and Algorithm 2, the latter has the practical benefit that one can directly work with a discrete tensor which can be more efficient than manipulating continuous functions. On the other hand, Algorithm 1 is closer to Chebfun's 


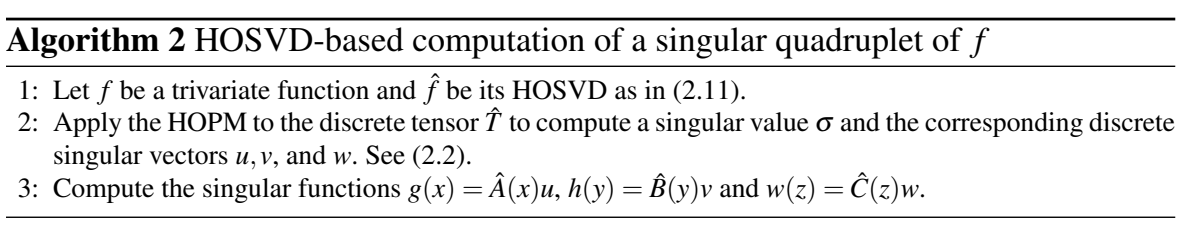

viewpoint of numerical computing with functions rather than discrete objects. It is mathematically more elegant in the sense that there is no discretizations involved. In addition, when a Tucker representation of $f$ as in (2.11) is unavailable, Algorithm 1 may be easier to implement. For example, a discretized HOPM can be applied; we illustrate this in Section 6.1, along with some of the practical difficulties.

We note that the goal of Algorithm 2 is not using HOSVD to approximate the function very accurately. Both Algorithms 1 and 2 solve the same problem of computing a singular quadruplet of $f$. It is standard to incorporate HOPM in greedy approximations as we later illustrate in experiments of Subsections 6.1.1 and 6.1.2. See also Subsection 6.1.3.

\section{The eigenvalue problem}

Eigenvalue problems are defined for matrices that are square. Analogously, for the eigenvalue problem to make sense, the domain of $f$ has to be a cube $[a, b]^{3}$.

Definition 3.1 $f(x, y, z)$ is called a cube function, if its domain is a cube, i.e., if $x, y$, and $z$ are all defined on a single interval $[a, b]$, i.e., $f:[a, b] \times[a, b] \times[a, b] \rightarrow \mathbb{R}$.

We first consider the case where $f$ is symmetric and then treat the general nonsymmetric case. Different types of eigenvalues are defined for a discrete tensor. Our definitions here correspond to the Z-eigenvalues of discrete tensors as in $[36,54]$. Such tensor eigenvalue problems have applications for example in random walks [3, 34], blind source separation [26] and MRI [48], and below we present its analogue in infinite dimensions for order three tensors.

\subsection{Symmetric functions}

Definition 3.2 A square function $f(x, y, z)$ is called symmetric if it is invariant under any permutation of variables, i.e., if $f(x, y, z)=f(y, x, z)=f(z, y, x)=f(x, z, y)$.

Definition 3.3 $(\lambda, g)$ is called an eigenpair of $f(x, y, z)$ if

$$
\left\{\begin{array}{l}
\int_{a}^{b} \int_{a}^{b} f(x, y, z) g(y) g(z) d z d y=\lambda g(x), \\
\int_{a}^{b} g(x)^{2} d x=1 .
\end{array}\right.
$$

Taking advantage of the symmetry results in the symmetric HOPM that is slightly cheaper but might not converge to an eigenvalue. This motivated Kolda and Mayo [29] to consider a shifted symmetric higher-order power method (SS-HOPM), which is 
guaranteed to converge to a stable eigenpair of discrete tensors of any order. Algorithm 3 is an extension of the SS-HOPM for trivariate functions. It uses a shift parameter $s$ whose appropriate choice guarantees convergence to an eigenvalue of $f$.

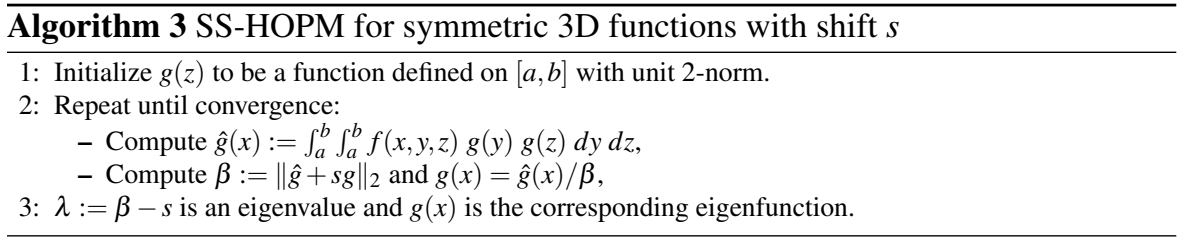

Let $f(x, y, z)$ be a symmetric chebfun3 of length $n$ and numerical Tucker rank $r$ and $g(x)$ be a 1D chebfun of length $n$. Similar to the discussion of the complexity of Algorithm 1, we can show that each iteration of the above continuous analogue of the SS-HOPM costs $\mathscr{O}\left(r^{3}+r n \log n\right)$ operations. Recall that the SS-HOPM for discrete full tensors of size $n \times n \times n$ requires $\mathscr{O}\left(n^{3}\right)$ arithmetic operations (as before, this reduces to $\mathscr{O}\left(r^{3}+r n\right)$ if a Tucker decomposition is available).

\subsection{Nonsymmetric functions}

A bivariate function defined on a square has left and right eigenfunctions which are not necessarily the same (unless the function is symmetric). Equivalently, the nonsymmetric 3D function $f(x, y, z)$ has modal eigenpairs, i.e., there are $x$-eigenpairs, $y$-eigenpairs, and $z$-eigenpairs.

Definition 3.4 $\left(\mu_{1}, g(x)\right)$ is called an $x$-eigenpair of $f(x, y, z)$ if

$$
\left\{\begin{array}{l}
\int_{a}^{b} \int_{a}^{b} f(x, y, z) g(y) g(z) d z d y=\mu_{1} g(x), \\
\int_{a}^{b} g(x)^{2} d x=1 .
\end{array}\right.
$$

A $y$-eigenpair $\left(\mu_{2}, h(y)\right)$ and a $z$-eigenpair $\left(\mu_{3}, w(z)\right)$ can be defined anaslogously. It is easy to see from (3.2) that $\left(\mu_{1}, g(x)\right)$ is an $x$-eigenpair if and only if $\left(-\mu_{1},-g(x)\right)$ is an $x$-eigenpair. (3.1) implies that the same relation holds for eigenpairs of symmetric functions.

\section{CP decomposition of trivariate functions}

Given a trivariate function $f(x, y, z)$, in this section we consider the problem of finding a CP decomposition (1.2). This is one of the canonical factorizations of a tensor of order $>2$. We note that the construction of Chebfun 3 [24] is based on the so-called Tucker decomposition, which is of a different nature. CP-type decompositions are explored in the literature as a tool to combat the curse of dimensionality for approximating multivariate functions, most notably in $[4,5]$. See also [8] for a least-square approach exploiting sparsity. The relation between the smoothness of an 
n-dimensional function $f \in L^{2}$ and the factors of its best approximation in both $\mathrm{CP}$ and Tucker formats is addressed in [60]. Let us emphasize also that our main problem in this paper is not function approximation. Our interest here is more on the connections between the spectral problem and CP decomposition for trivariate functions. Even though our results can perhaps be extended to higher dimensional functions, we have focused on 3D functions for which Chebfun3 is available as a convenient exploratory numerical environment.

Generally, computing the CP decomposition of a discrete tensor is NP-hard. For example, an $n \times n \times n$ tensor can have a CP rank $\mathscr{O}\left(n^{2}\right)$ : a trivial upper bound is $n^{2}$, and the bound $2\left\lceil\frac{n^{3}}{3 n-2}\right\rceil \approx \frac{2}{3} n^{2}$ is given in [6, Ex. 12], building upon [32,35,53]. To obtain a computationally tractable algorithm for CP decomposition of trivariate functions, we shall invoke a key tool in the theory of computing the $\mathrm{CP}$ decomposition of a three-way tensor. Namely, it is sufficient for the direct computation of the $\mathrm{CP}$ decomposition

$$
T=\sum_{\ell=1}^{r} \mathbf{a}_{\ell} \circ \mathbf{b}_{\ell} \circ \mathbf{c}_{\ell},
$$

of a discrete tensor $T$ of size $m \times n \times \ell$ that the following two conditions are satisfied: (i) $r \leq \min (m, n, \ell)$ (or a slightly weaker condition), and (ii) two of the matrices $A=\left[\mathbf{a}_{1}, \ldots, \mathbf{a}_{r}\right], B=\left[\mathbf{b}_{1}, \ldots, \mathbf{b}_{r}\right]$ and $C=\left[\mathbf{c}_{1}, \ldots, \mathbf{c}_{r}\right]$ have full column rank. Specifically, the $\mathrm{CP}$ can be computed exactly via a matrix decomposition, namely a simultaneous diagonalization of the slices, which in turn can be computed via a generalized eigenvalue problem.

The essence is that two random slices will have a generalized eigenvalue decomposition where the matrices of left and right eigenvector have columns equal to the factors in the CP decomposition. This fact was reported in [46], and independently in [33], and this idea has been refined and generalized in [10,13]. In [16] it is shown that the CP decomposition can be computed using standard linear algebra provided that Kruskal's condition [31] (which guarantees uniqueness of CP decompositon) is satisfied. Since the algorithms in these recent papers are rather intricate, here we consider the continuous analogues of the classical result [33],[46].

This result has remained of relatively limited applicability in the context of discrete tensors, primarily due to the condition " $\mathrm{CP}$ rank $r \leq \min (m, n, \ell)$ " being too restrictive. However, consider our main subject of trivariate functions. Here, the dimension, if any, is $\infty \times \infty \times \infty$. Crucially, this means that the above requirement reduces merely to that the trivariate function is of finite rank. This is not much of a restriction, since in practice, we usually work with functions of finite rank (this is surely the case in Chebfun3). This suggests that perhaps for a wide class of trivariate functions of practical interest, the $\mathrm{CP}$ decomposition could be computed via linear algebra algorithms, provided that the linear independence condition holds among the $\mathrm{CP}$ factors (this does pose a practical limitation in the applicability).

We describe the process for trivariate functions. Let us introduce the factor quasimatrices $\mathbf{G}(x)=\left[g_{1}(x), \ldots, g_{r}(x)\right] \in \mathbb{R}^{\infty \times r}$ (here $\infty$ represents the interval $\left.[a, b]\right), \mathbf{H}(y)=$ $\left[h_{1}(y), \ldots, h_{r}(y)\right] \in \mathbb{R}^{\infty \times r}(\infty$ represents $[c, d])$, and $\mathbf{W}(z)=\left[w_{1}(z), \ldots, w_{r}(z)\right] \in \mathbb{R}^{\infty \times r}$, in which $\infty$ represents $[e, l]$. Then, we can write $f$ in (1.2) as

$$
C \times{ }_{1} \mathbf{G}(x) \times{ }_{2} \mathbf{H}(y) \times{ }_{3} \mathbf{W}(z),
$$


where $C$ is an $r \times r \times r$ diagonal core tensor with $i$ th diagonal element $\alpha_{i}$ and zero non-diagonal entries.

We now assume that $\mathbf{G}(x)$ and $\mathbf{H}(y)$ have full column rank $r$, i.e., $g_{1}, \ldots, g_{r}$ are linearly independent in $L^{2}([a, b])$ and $h_{1}, \ldots, h_{r}$ are linearly independent in $L^{2}([c, d])$. The $z=z_{0}$ slice $f\left(x, y, z_{0}\right)$, for any $z_{0} \in[e, l]$, is the bivariate function

$$
f\left(x, y, z_{0}\right)=\mathbf{H}(y)\left[\begin{array}{llll}
\alpha_{1} w_{1}\left(z_{0}\right) & & & \\
& \alpha_{2} w_{2}\left(z_{0}\right) & & \\
& & \ddots & \\
& & & \alpha_{r} w_{r}\left(z_{0}\right)
\end{array}\right] \mathbf{G}(x)^{T}
$$

Another slice $z=z_{1}$ gives

$$
f\left(x, y, z_{1}\right)=\mathbf{H}(y)\left[\begin{array}{llll}
\alpha_{1} w_{1}\left(z_{1}\right) & & & \\
& \alpha_{2} w_{2}\left(z_{1}\right) & & \\
& & \ddots & \\
& & & \alpha_{r} w_{r}\left(z_{1}\right)
\end{array}\right] \mathbf{G}(x)^{T} .
$$

From these we see that $\left(f\left(x, y, z_{0}\right), f\left(x, y, z_{1}\right)\right)$, regarded as a continuous analogue of matrix pairs, has the eigenvalue equations

$$
f\left(x, y, z_{1}\right)\left(\mathbf{G}(x)^{T}\right)^{\dagger}=f\left(x, y, z_{0}\right)\left(\mathbf{G}(x)^{T}\right)^{\dagger} \operatorname{diag}\left(\frac{w_{1}\left(z_{1}\right)}{w_{1}\left(z_{0}\right)}, \ldots, \frac{w_{r}\left(z_{1}\right)}{w_{r}\left(z_{0}\right)}\right) .
$$

Here $\left(\mathbf{G}(x)^{T}\right)^{\dagger} \in \mathbb{R}^{\infty \times r}$ is the quasimatrix pseudoinverse of $\mathbf{G}(x)^{T}$ such that $\mathbf{G}(x)^{T}\left(\mathbf{G}(x)^{T}\right)^{\dagger}=$ $I_{r}$ and $\sigma_{i}\left(\left(\mathbf{G}(x)^{T}\right)^{\dagger}\right)=1 / \sigma_{i}(\mathbf{G}(x))$. The pair $\left(f\left(x, y, z_{0}\right), f\left(x, y, z_{1}\right)\right)$ also has the analogous left eigenvalue equation

$$
\mathbf{H}(y)^{\dagger} f\left(x, y, z_{1}\right)=\operatorname{diag}\left(\frac{w_{1}\left(z_{1}\right)}{w_{1}\left(z_{0}\right)}, \ldots, \frac{w_{r}\left(z_{1}\right)}{w_{r}\left(z_{0}\right)}\right) \mathbf{H}(y)^{\dagger} f\left(x, y, z_{0}\right) .
$$

As in standard matrix theory $[14, \S .4 .5]$, the equations (4.3) and (4.4) also imply the simultaneous diagonalization property

$$
\begin{aligned}
\mathbf{H}(y)^{\dagger} f\left(x, y, z_{0}\right)\left(\mathbf{G}(x)^{T}\right)^{\dagger} & =\left[\begin{array}{lll}
\alpha_{1} w_{1}\left(z_{0}\right) & & \\
& \ddots & \\
& & \alpha_{r} w_{r}\left(z_{0}\right)
\end{array}\right], \\
& \mathbf{H}(y)^{\dagger} f\left(x, y, z_{1}\right)\left(\mathbf{G}(x)^{T}\right)^{\dagger}=\left[\begin{array}{lll}
\alpha_{1} w_{1}\left(z_{1}\right) & & \\
& \ddots & \\
& & \alpha_{r} w_{r}\left(z_{1}\right)
\end{array}\right] .
\end{aligned}
$$

In order to obtain the decomposition (4.7) and hence the desired factors $\mathbf{G}(x)$ and $\mathbf{H}(y)$ in practice, we reduce the task to a matrix eigenvalue problem. First, compute the SVD of the bivariate functions

$$
f\left(x, y, z_{0}\right)=\mathbf{U}_{0}(y) S_{0} \mathbf{V}_{0}(x)^{T}, \quad f\left(x, y, z_{1}\right)=\mathbf{U}_{1}(y) S_{1} \mathbf{V}_{1}(x)^{T} .
$$


By (4.3) and (4.4), unless $w_{i}\left(z_{j}\right)=0$ for some $(i, j), \mathbf{U}_{0}$ and $\mathbf{U}_{1}$ have the same column space spanned by those of $\mathbf{H}(y)$. In practice, we compute the numerical column space by finding the nonnegligible singular values larger than a tolerance, and find the corresponding columns $\hat{\mathbf{U}}_{0}(y)$ of $\mathbf{U}_{0}(y)$ (its first $\hat{r}$ columns). We do the same to the row space by extracting the first $\hat{r}$ rows $\hat{\mathbf{V}}_{0}(x)^{T}$ of $\mathbf{V}_{0}(x)^{T}$, and project both $f\left(x, y, z_{0}\right)$ and $f\left(x, y, z_{1}\right)$ onto the spaces to obtain two matrices of size $\hat{r} \times \hat{r}$ :

$$
\begin{aligned}
& A_{0}=\hat{\mathbf{U}}_{0}(y)^{T} f\left(x, y, z_{0}\right) \hat{\mathbf{V}}_{0}(x)=\left(\hat{\mathbf{U}}_{0}(y)^{T} \mathbf{U}_{0}(y)\right) S_{0}\left(\mathbf{V}_{0}(x)^{T} \hat{\mathbf{V}}_{0}(x)\right), \\
& A_{1}=\hat{\mathbf{U}}_{0}(y)^{T} f\left(x, y, z_{1}\right) \hat{\mathbf{V}}_{0}(x)=\left(\hat{\mathbf{U}}_{0}(y)^{T} \mathbf{U}_{1}(y)\right) S_{1}\left(\mathbf{V}_{1}(x)^{T} \hat{\mathbf{V}}_{0}(x)\right) .
\end{aligned}
$$

One then finds the generalized eigenvalue decomposition for the matrix pair $\left(A_{0}, A_{1}\right)$ as

$$
Y^{T} A_{0} X=\Lambda_{0}, \quad Y^{T} A_{1} X=\Lambda_{1}
$$

where $\Lambda_{0}, \Lambda_{1}$ are diagonal matrices. This can be done by the standard QZ algorithm [39] for generalized eigenvalue problems, which is backward stable. We then obtain the right eigenfunctions in (4.5), (4.6) as $\left(\hat{\mathbf{G}}(x)^{T}\right)^{(\dagger)}:=\hat{\mathbf{V}}_{0}(x) X$ (which approximates $\left.\left(\mathbf{G}(x)^{T}\right)^{\dagger}\right)$ and the left eigenfunctions as $\hat{\mathbf{H}}(y)^{(\dagger)}:=Y^{T} \hat{\mathbf{U}}_{0}(y)^{T}$ (which approximates $\left.\mathbf{H}(y)^{\dagger}\right)$. We will also use approximants $\hat{\mathbf{G}}(x), \hat{\mathbf{H}}(y)$ to $\mathbf{G}(x), \mathbf{H}(y)$, which are obtained as

$$
\hat{\mathbf{G}}(x)=\hat{\mathbf{V}}_{0}(x) X^{-T}, \quad \hat{\mathbf{H}}(y)=\hat{\mathbf{U}}_{0}(y) Y^{-T} .
$$

Finally, to obtain an approximant to $\mathbf{W}(z)$, we see from (4.2) that

$$
c_{i} w_{i}(z)=\int_{a}^{b} \int_{c}^{d} f(x, y, z) g_{i}^{\dagger}(x) h_{i}^{\dagger}(y) d y d x .
$$

Here $g_{i}^{\dagger}(x), h_{i}^{\dagger}(y)$ are the $i$ th column of the quasimatrices $\mathbf{G}(x)^{\dagger}, \mathbf{H}(y)^{\dagger}$, which we approximate via those of $\hat{\mathbf{G}}(x)^{(\dagger)}, \hat{\mathbf{H}}(y)^{(\dagger)}$. We can retrieve $c_{i}, w_{i}(z)$ from (4.12) simply by the normalization $\left\|w_{i}(z)\right\|=1$, that is, $c_{i}=\left\|\int_{a}^{b} \int_{c}^{d} f(x, y, z) g_{i}(x) h_{i}(y) d y d x\right\|, w_{i}(z)=$ $\frac{1}{c_{i}} \int_{a}^{b} \int_{c}^{d} f(x, y, z) g_{i}(x) h_{i}(y) d y d x$. Putting these together, we obtain an approximate CP decomposition $f \approx \sum_{i=1}^{r} \alpha_{i} \hat{g}_{i}(x) \hat{h}_{i}(y) \hat{w}_{i}(z)$. We summarize the process in Algorithm 4.

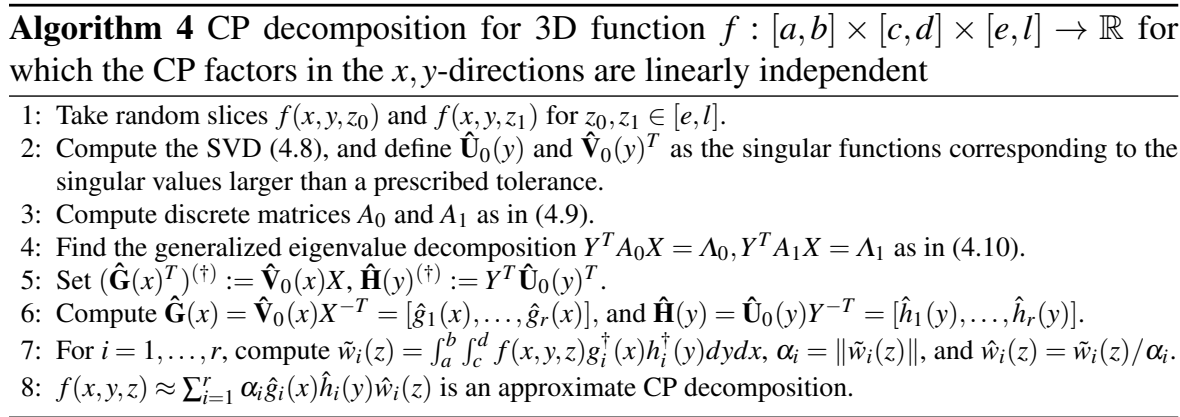

For improved reliability, instead of starting from two specific slices (4.3) and (4.4), it is recommended that one take a number of randomly sampled slices and take 
their average (or integrate with respect to two different weight functions). Then the diagonal matrices in (4.3), (4.4) are modified accordingly, but the rest of the argument remains the same. This avoids the defective case where $w_{i}\left(z_{j}\right)=0$ for $j=0,1$, for some $i$ (in which case the corresponding elements $h, g$ cannot be retrieved).

The success of Algorithm 4 can be measured by $\left\|f(x, y, z)-\sum_{i=1}^{r} \alpha_{i} \hat{g}_{i}(x) \hat{h}_{i}(y) \hat{w}_{i}(z)\right\|$, which is hoped to be sufficiently small. Failure of this can be caused (and predicted within the algorithm) by the condition numbers of $\hat{\mathbf{G}}(x)$ and $\hat{\mathbf{H}}(y)$, and by examining whether $\mathbf{H}(y)^{\dagger} f\left(x, y, z_{2}\right)\left(\mathbf{G}(x)^{T}\right)^{\dagger}$ is close to diagonal for another random slice $f\left(x, y, z_{2}\right)$.

When $\mathbf{H}$ or $\mathbf{G}$ is rank deficient, Algorithm 4 will break down; numerically this means the matrices $X$ and $Y$ become highly ill-conditioned. If two factors among $\mathbf{H}$, $\mathbf{G}$ and $\mathbf{W}$ have full column rank, then we can still compute the $\mathrm{CP}$ decomposition by working e.g. with $g(x, y, z)=f(y, z, x)$. If two or more of the three quasimatrices are rank-deficient, then this remedy will still not help. Computing the $\mathrm{CP}$ in such cases is left an open problem-algorithms that are applicable under relaxed conditions have been proposed for discrete tensors, e.g. in [10] and [16], but the algorithm is expensive and its continuous analogue appears to be nontrivial.

\section{Two-way odeco functions and decay of CP values}

In this section we explore the CP-values of trivariate functions. For bivariate functions, the singular values decay geometrically if the function is analytic in the domain. We suspect that an analogous result might hold for the trivariate case; here we give a partial answer by proving the same holds for CP-values of trivariate functions possessing a property called two-way odeco, which is a natural relaxation of odeco tensors/functions. Odeco tensors were introduced in [63], see also [27] for its analysis.

Definition 5.1 $f(x, y, z)$ is called orthogonally decomposable, or odeco, if it can be written in the polyadic form (1.2) such that the functions $g_{j}(x), h_{j}(y)$, and $w_{j}(z)$ are orthogonal, i.e., if

$$
\int_{a}^{b} g_{i}(x) g_{j}(x) d x=\int_{c}^{d} h_{i}(y) h_{j}(y) d y=\int_{e}^{l} w_{i}(z) w_{j}(z) d z=\delta_{i, j},
$$

for every fixed $i, j=1,2, \cdots, f(x, y, z)$ is called two-way odeco, if two of the set of functions $g_{j}(x), h_{j}(y)$, and $w_{j}(z)$ are orthogonal.

Suppose that we know the CP decomposition of $f$, and the factors may or may not be orthonormal. Note that the $\mathrm{CP}$ decomposition of $f$ is a special case of the Tucker format in which the discrete core tensor is diagonal. Now consider converting the $\mathrm{CP}$ to an odeco expansion of $f$. To do so, we can always orthonormalize factors $g_{j}(x)$, $h_{j}(y)$ and $w_{j}(z)$ of the $\mathrm{CP}$ decomposition of $f$ e.g., using the QR decomposition [58]. In order to replace the factors with the new orthonormalized factors, the diagonal core tensor has to be multiplied by three discrete matrices which are triangular; hence the core tensor will not necessarily be kept diagonal. A sufficient condition for the core tensor to be kept diagonal is that the given function $f$ is originally odeco in 
which case the matrices $R$ from the QR factorizations are actually diagonal. In this case, every term in the CP expansion of $f$ is a singular quadruplet of $f$; this can be seen straightforwardly by verifying (2.7). In other words, for an odeco function, the triples $\left(g_{j}(x), h_{j}(y), w_{j}(z)\right)$ corresponding to the rank-1 terms are singular function tuples and the corresponding coefficient is a singular value ${ }^{3}$. The converse is not true-singular quadruplets are not necessarily $\mathrm{CP}$ quadruplets; we return to this in Section 5.2.

For odeco trivariate functions, Algorithm 4 can always compute the $\mathrm{CP}$ decomposition: the linearly independence assumption is trivially satisfied because the three quasimatrices $\mathbf{G}(x), \mathbf{H}(y), \mathbf{W}(z)$ are all orthonormal, hence have full column rank $r$. This also gives an algorithm to check whether a trivariate function is odeco: use Algorithm 4 to compute the CP decomposition, and check if the quasimatrices $\mathbf{G}(x)$, $\mathbf{H}(y), \mathbf{W}(z)$ are all orthonormal. The function is not odeco if Algorithm 4 fails or one of the quasimatrices is not orthonormal.

Clearly, two-way odeco is a more general class of trivariate functions than odeco (although admittedly still a very special class). In this section we show that the CP values decay geometrically for two-way odeco analytic functions, and therefore be approximated by a finite-rank tensor with exponential accuracy.

Unless otherwise mentioned, in this section we suppose $f$ is two-way odeco (in $y, z$ directions, for definiteness). That is, we assume $f$ can be written as

$$
f(x, y, z)=\sum_{i=1}^{\infty} \alpha_{i} g_{i}(x) h_{i}(y) w_{i}(z)
$$

with $g_{i}, h_{i}, w_{i}$ all real-valued, having unit norms (in particular $\left\|g_{i}\right\|=1$ for every $i \in \mathbb{N}$ ), $\left\langle h_{i}, h_{j}\right\rangle=\left\langle w_{i}, w_{j}\right\rangle=\delta_{i j}$ (in the $L^{2}$ inner products $\left\langle h_{i}, h_{j}\right\rangle=\int_{c}^{d} h_{i}(y) h_{j}(y) d y$ and $\left.\left\langle w_{i}, w_{j}\right\rangle=\int_{e}^{l} w_{i}(z) w_{j}(z) d z\right)$, and $\alpha_{1} \geq \alpha_{2} \geq \alpha_{3} \geq \cdots \geq 0$; there is no loss of generality in assuming positivity.

\subsection{Geometric decay of CP values for two-way odeco functions}

Here we show for two-way odeco functions that the CP-values decay geometrically. The analysis below closely follows that in [37] for bivariate functions, but with some modifications to handle the trivariate case (e.g. the $\sqrt{r+1}$ factor).

Lemma 5.1 Let $f:[a, b] \times[c, d] \times[e, l] \rightarrow \mathbb{R}$ be a trivariate function that is two-way odeco (in $y, z$ ) with $C P$-values $\alpha_{1} \geq \alpha_{2} \geq \cdots$ as in (5.1). Then for any trivariate function of the form

$$
\hat{f}(x, y, z):=\sum_{j=1}^{r} s_{j}(y) p_{j}(x, z)
$$

we have

$$
\alpha_{r+1} \leq \sqrt{r+1}\|f-\hat{f}\|
$$

\footnotetext{
${ }^{3}$ See e.g. [54] for an analogous discussion regarding eigenvalues of symmetric discrete tensors.
} 
Proof Since $\left[h_{1}, \ldots, h_{r+1}\right]$ spans a subspace of dimension $r+1$, we can choose

$$
\phi(y)=\sum_{j=1}^{r+1} c_{j} h_{j}(y)
$$

so that $\|\phi\|=1$ and $\hat{f} \times_{2} \phi:=\int_{c}^{d} \hat{f}(x, y, z) \phi(y) d y=0$; specifically, we choose $\left[c_{1}, \ldots, c_{r+1}\right]$ so that it has unit norm and $\int_{c}^{d} \phi(y) s_{j}(y) d y=0$ for $j=1, \ldots, r$ : more precisely, we can take $\left[c_{1}, \ldots, c_{r+1}\right]^{T}$ to be a null vector of the $r \times(r+1)$ matrix with $(i, j)$ element $\left\langle s_{i}, h_{j}\right\rangle$. Therefore

$$
\left\|(f-\hat{f}) \times_{2} \phi\right\|=\left\|f \times_{2} \sum_{j=1}^{r+1} c_{j} h_{j}\right\|=\left\|\sum_{j=1}^{r+1} c_{j} \alpha_{j} g_{j}(x) w_{j}(z)\right\|=:\|G(x, z)\| .
$$

For each $\ell \in\{1, \ldots, r+1\}$, we have

$$
G(x, z) \times_{1} g_{\ell}(x)=\sum_{j=1}^{r+1} c_{j} \alpha_{j}\left\langle g_{\ell}, g_{j}\right\rangle w_{j}(z) .
$$

Now let $\ell_{*}=\operatorname{argmax}_{1 \leq j \leq r+1}\left|c_{j}\right|$. Then we have $\left|c_{\ell_{*}}\right| \geq \frac{1}{\sqrt{r+1}}$, since $\|c\|=1$. Taking $\ell=\ell_{*}$ in the above equation and integrating with $w_{\ell_{*}}(z)$ we obtain

$$
\left|\left(G(x, z) \times{ }_{1} g_{\ell_{*}}(x)\right) \times{ }_{3} w_{\ell_{*}}(z)\right|=\left|c_{\ell_{*}} \alpha_{\ell_{*}}\left\langle g_{\ell_{*}}, g_{\ell_{*}}\right\rangle\right| \geq \frac{\alpha_{\ell_{*}}}{\sqrt{r+1}},
$$

where we used the fact $\left\|g_{\ell_{*}}\right\|=1$. Recall that in the line following (5.1) we assumed, without loss of generality, that all the CP factors have unit norms. Thus $\mid(f-\hat{f}) \times_{1}$ $g_{\ell_{*}} \times_{2} \phi \times_{3} w_{\ell_{*}} \mid \geq \frac{\alpha_{\ell_{*}}}{\sqrt{r+1}} \geq \frac{\alpha_{r+1}}{\sqrt{r+1}}$ with $\|\phi\|=\left\|w_{\ell_{*}}\right\|=1$, establishing (5.2).

Now we establish the geometric decay of $\mathrm{CP}$ values. In the following theorem, $E_{\rho}$ denotes an open Bernstein ellipse with foci at \pm 1 and semiminor and semimajor axis lengths summing to $\rho>1$. Specifically, $E_{\rho}:=\left\{\frac{1}{2}\left(\rho z+(\rho z)^{-1}\right):|z|<1, z \in \mathbb{C}\right\}$.

Theorem 5.1 Let $f(x, y, z):[-1,1]^{3} \rightarrow \mathbb{R}$ be two-way odeco in $y, z$, and for every fixed $x_{0}, z_{0} \in[-1,1]^{2}$, suppose that the univariate function $f\left(x_{0}, y, z_{0}\right)$ is analytic in $[-1,1]$ and analytically continuable to the open Bernstein ellipse $E_{\rho}$ where it is uniformly bounded by $M$. Then the CP-values of $f$ decay as

$$
\alpha_{r+1} \leq \frac{2 M \sqrt{r+1}}{\rho-1} \rho^{-r}
$$

Proof Write $f$ in Chebyshev expansion with respect to $y$ as

$$
f(x, y, z)=\frac{a_{0}(x, z)}{2}+\sum_{j=1}^{\infty} a_{j}(x, z) T_{j}(y) .
$$

Specifically, we have

$$
a_{j}(x, z)=\frac{1}{\pi} \int_{-\pi}^{\pi} f(x, \cos \theta, z) e^{\mathrm{i} j \theta} d \theta .
$$


The imposed analyticity assumptions guarantee that [59, Thm. 8.1] $\left|a_{j}(x, z)\right| \leq 2 M \rho^{-j}$, uniformly in $x \in[a, b]$ and $z \in[e, l]$. By taking

$$
\hat{f}(x, y, z):=\frac{a_{0}(x, z)}{2}+\sum_{j=1}^{r} a_{j}(x, z) T_{j}(y),
$$

together with (5.2) we obtain

$$
\alpha_{r+1} \leq \sqrt{r+1}\|f-\hat{f}\|=\sqrt{r+1}\left\|\sum_{j=r+1}^{\infty} a_{j}(x, z) T_{j}(y)\right\| \leq \frac{2 M \sqrt{r+1}}{\rho-1} \rho^{-r}
$$

as required.

If $f$ is entire and (two-way) odeco, then the decay of the CP-values is even faster, i.e., super-geometric. Moreover, employing an appropriate change of variables, we can establish the geometric decay to the case where the domain of $f$ is a general rectangular parallelepiped $[a, b] \times[c, d] \times[e, l]$.

In the case that $f$ is only $v$ times differentiable, we can prove the decay rate of $\mathrm{CP}$ values to be algebraic, like $r^{-(v+1)}$ by invoking Theorem 7.1 instead of 8.1 in [59].

While two-way odeco functions are more general than odeco functions, a slightly wider class (for discrete tensors) is treated in [62, Def. 3.5], called functions admitting a strong two-orthogonal decomposition, to identify a sufficient condition for a Schmidt-Eckart-Young decomposition (a decomposition for which extracting the leading $k$ terms gives the best rank- $k$ approximation for all $k$ ) to exist. For example, a function that is not two-way odeco but has a (continuous analogue of the) strong twoorthogonal decomposition is $g_{1}(x) h_{1}(y) w_{1}(z)+g_{2}(x) h_{2}(y) w_{2}(z)+g_{3}(x) h_{3}(y) w_{3}(z)$, where $\left[w_{1}, w_{2}, w_{3}\right]$ is orthogonal and so are $\left\langle g_{1}, g_{2}\right\rangle=\left\langle g_{1}, g_{3}\right\rangle=\left\langle h_{2}, h_{3}\right\rangle=0$, but otherwise arbitrary, in particular $\left\langle g_{2}, g_{3}\right\rangle \neq 0$. Examining whether the geometric decay of the $\mathrm{CP}$-values extends to the strong two-orthogonal case or to more general trivariate functions is left as an open problem.

\subsection{Low-rank approximation via CP for two-way odeco functions}

We have derived decay bounds for CP-values. We next explore the best rank- $r$ approximation to $f$, along with the connection between the CP decomposition and singular quadruplets.

Proposition 5.1 Let $f(x, y, z)$ be a two-way odeco function (in $y, z$ as in the $C P$ decomposition (5.1)). Then the following hold:

1. For every $r \in \mathbb{N}, f_{r}:=\sum_{i=1}^{r} \alpha_{i} g_{i}(x) h_{i}(y) w_{i}(z)$ is the best rank-r approximant of $f$.

2. For every $i \in \mathbb{N},\left(\alpha_{i}, g_{i}, h_{i}, w_{i}\right)$ is a singular quadruplet satisfying (2.7), and $\left(\alpha_{1}, g_{1}, h_{1}, w_{1}\right)$ is the largest singular quadruplet of $f$.

3. For any $\hat{f}$ of $C P$-rank $r,\|f-\hat{f}\| \geq \alpha_{r+1}$, with equality attained when $\hat{f}=f_{r}$.

4. If $f$ further satisfies the assumptions in Theorem 5.1, it can be approximated by a rank-r function with exponential accuracy, namely $\left\|f-f_{r}\right\| \leq \frac{2 M \sqrt{r+1}}{\rho-1} \rho^{-r}$. 
Proof The proof of the first statement is a straightforward adaptation of [62, Thm. 3.6]: We can obviously rewrite $f$ as

$$
f(x, y, z)=\sum_{i=1}^{\infty} \alpha_{i} G_{i}(x, y) w_{i}(z)
$$

where $G_{i}(x, y):=g_{i}(x) h_{i}(y)$ is a bivariate function (of rank one). Consider the Euclidean inner product of bivariate functions (see the definition of the tensor space isomorphism and the following discussion in [23, pp. 59-61])

$$
\langle G(x, y), H(x, y)\rangle:=\int_{a}^{b} \int_{c}^{d} G(x, y) H(x, y) d y d x .
$$

Note that (5.6) is the SVD of $f$ regarded as a bivariate function of $z$ from one side and $(x, y)$ form the other, because

$$
\begin{aligned}
\left\langle G_{i}(x, y), G_{j}(x, y)\right\rangle & =\int_{a}^{b} \int_{c}^{d} g_{i}(x) h_{i}(y) g_{j}(x) h_{j}(y) d y d x \\
& =\left\langle g_{i}(x), g_{j}(x)\right\rangle\left\langle h_{i}(y), h_{j}(y)\right\rangle \\
& =\delta_{i j}
\end{aligned}
$$

where the last equality relies on the fact that $\left\|g_{i}\right\|=1$ for every $i \in \mathbb{N}$. Now since the SVD provides an optimal truncation of bivariate functions, the same holds for (5.1), i.e., $\sum_{i=1}^{r} \alpha_{i} g_{i}(x) h_{i}(y) w_{i}(z)$ is the best rank- $r$ approximation of $f$.

We next treat the second statement. It is straightforward to verify that $i \in \mathbb{N}$, $\left(\alpha_{i}, g_{i}, h_{i}, w_{i}\right)$ satisfies (2.7). To see that $\left(\alpha_{1}, g_{1}, h_{1}, w_{1}\right)$ is the largest singular quadruplet of $f$, let $g(x)$ be a univariate function of unit norm and consider

$$
f_{1}(y, z):=\int_{a}^{b} f(x, y, z) g(x) d x=\left\langle g_{1}, g\right\rangle \alpha_{1} h_{1}(y) w_{1}(z)+\sum_{i=2}^{\infty}\left\langle g_{i}, g\right\rangle \alpha_{i} h_{i}(y) w_{i}(z) .
$$

By the orthonormality assumption on $h_{i}$ and $w_{i}$, we see that the largest singular value of $f_{1}(y, z)$ is the largest value among $\left\langle g_{i}, g\right\rangle \alpha_{i}$ for $i=1,2, \ldots$. By the normalization this is easily seen to be bounded by $\alpha_{1}$. Since this holds for any $g(x)$, it follows that $\alpha_{1}$ is an upper bound for the largest singular value of $f$. Furthermore, equality holds by taking $g(x):=g_{1}(x)$, so $\left(\alpha_{1}, g_{1}, h_{1}, w_{1}\right)$ is the largest singular quadruplet of $f$.

The third statement is a trivial consequence of the first two. The fourth follows directly from combining the third statement and (5.4).

This result tells us that in order to compute the best rank- $r$ approximant to $f$ we can either truncate the leading CP terms, or successively find and subtract the best rank-1 approximant. Another consequence of this result is that if we perturb a rank1 function $f$ by a function that is orthogonal to $f$ in two ways, then its best rank-1 approximant remains intact (until the perturbation is large enough to change the first term in the CP decomposition).

As the proof suggests, the first statement still holds if we relax the assumption on $f$ to having a strong two-orthogonal decomposition.

Regarding the second statement of Proposition 5.1, one might suspect that the converse could also hold: every singular quadruplet of $f$ would be a CP quadruplet, 
i.e., CP and SVD are identical. It turns out that this is not true, even for (three-way) odeco functions. For example, consider an odeco function $f=\sigma_{1} g_{1} h_{1} w_{1}+\sigma_{2} g_{2} h_{2} w_{2}$, where $\sigma_{1}, \sigma_{2}>0$ and $\left\langle g_{1}, g_{2}\right\rangle=\left\langle h_{1}, h_{2}\right\rangle=\left\langle w_{1}, w_{2}\right\rangle=0$. Note that this is the unique $\mathrm{CP}$ decomposition of $f$ by Kruskal's sufficient condition [31]. However, $f$ has other singular quadruplets: Let $s, t \in \mathbb{R}$ be such that $s^{2}+t^{2}=1$ and $s \sigma_{1}=t \sigma_{2}$, and define $\tilde{g}=s g_{1}+t g_{2}, \tilde{h}=s h_{1}+t h_{2}, \tilde{w}=s w_{1}+t w_{2}$, and $\tilde{\sigma}=s \sigma_{1}$. Then we can verify that $(\tilde{\sigma}, \tilde{g}, \tilde{h}, \tilde{w})$ is a singular quadruplet, satisfying $(2.7)^{4}$. In any case, by the first statement of Proposition 5.1, the largest singular quadruplet is a CP quadruplet.

The above example, together with the second statement of Proposition 5.1, shows that the $\mathrm{CP}$ quadruplets are a strict subset of the singular quadruplets for (two-way) odeco functions. However, in view of the first statement of Proposition 5.1, we expect the existence of singular quadruplets that are not $\mathrm{CP}$ quadruplets to be of marginal significance, at least in the context of approximating $f$ by a low-rank function.

We close this section by a discussion of possible extensions to functions of general dimension $d \geq 3$. It seems possible to extend the variational formulation of spectral notions like singular values and eigenvalues and all our algorithms to higher dimensions. On the other hand, our arguments on the decay of CP-values and singular values seem to work in higher dimensions without fundamentally different assumptions being necessary. For instance, we do not need to assume that the function is odeco in $d-1$ variables; two-way odeco is enough for any dimension $d \geq 3$. The crucial necessity is to be able to use a splitting of variables in two subsets so that one of the factors of $\mathrm{CP}$ decomposition in each subset is orthogonal. We leave a rigorous formulation and the corresponding decay rates for future research. Note that higher dimensional extensions of Algorithm 4 (cf. [33, sec. 6], more generally [10]) can be used to check whether a given function is odeco. See the beginning of this section.

\section{Numerical examples}

Unless otherwise stated, 3D functions in this section are defined over $[-1,1]^{3}$. All the experiments were done in MATLAB building upon the Chebfun package [17].

\subsection{Best rank-1 approximation}

By the power method we can find the largest singular quadruplet, which by Theorem 2.1, gives the best rank-1 approximant to $f$. Figure 6.1 (left) shows the convergence history of power method applied to the function $f(x, y, z)=\exp (\sin (x+y+z))$, measured by the error of the Rayleigh functional $|\hat{\sigma}-\sigma|$ (where we take the 'exact' value $\sigma$ to be the final value of the iterate) and the residual, computed by (recalling (2.7))

$$
\left\|\left[\begin{array}{l}
\int_{c}^{d} \int_{e}^{l} f(x, y, z) \hat{w}(z) \hat{h}(y) d z d y-\hat{\sigma} \hat{g}(x) \\
\int_{a}^{b} \int_{e}^{l} f(x, y, z) \hat{w}(z) \hat{g}(x) d z d x-\hat{\sigma} \hat{h}(y) \\
\int_{a}^{b} \int_{c}^{d} f(x, y, z) \hat{h}(y) \hat{g}(x) d y d x-\hat{\sigma} \hat{w}(z)
\end{array}\right]\right\|_{2}
$$

\footnotetext{
${ }^{4}$ The singular quadruplets for odeco tensors is fully studied in [45], which shows that the number of
} 
where the values wearing a hat indicate computed quantities. Here the norm is the standard $\ell_{2}$ norm.

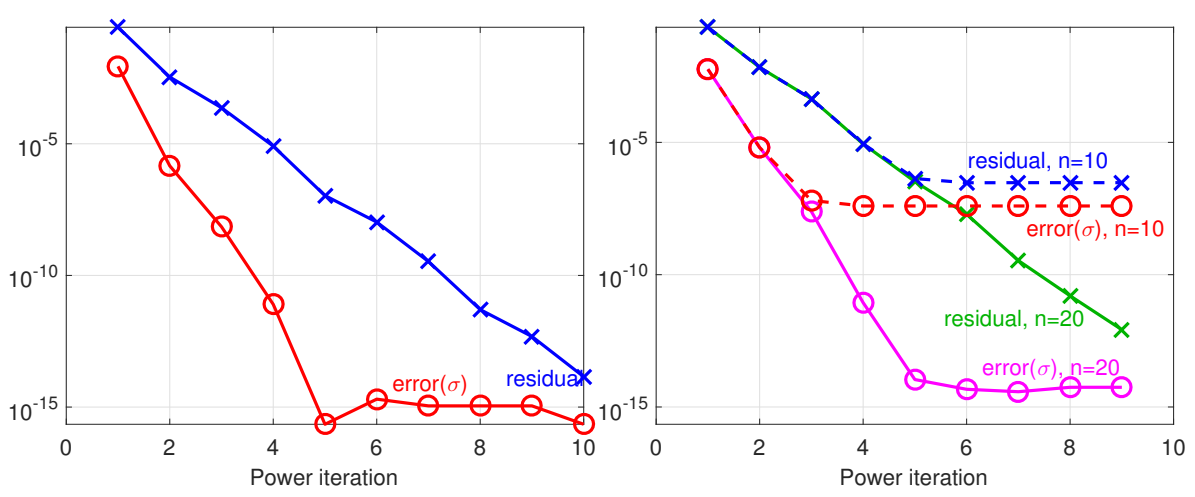

Fig. 6.1 Left: Typical convergence of power method (Algorithm 1, or equivalently 2) for the largest singular quadruplet of $f(x, y, z)=\exp (\sin (x+y+z))$. Right: convergence of power method with $n \times n \times n$ discretized tensors, for $n=10$ and $n=20$.

Observe that the error of the Rayleigh quotient scales like the squared of the norm of the residual; this is a known phenomenon [63]. Since this function is not twoway odeco, the $\mathrm{CP}$ and singular value decompositions are not identical, in particular, $\sigma_{1} \neq \alpha_{1}$

To illustrate the difference between continuous and discrete HOPM, Figure 6.1 (right) shows the convergence obtained as follows: discretize the function in a $n \times n \times$ $n$ Chebyshev grid, and apply a "weighted" HOPM, where the weights are those in the Clenshaw-Curtis quadrature [59, Chap. 19]. We then convert the computed discrete rank-one tensor to a continuous rank-one function via Chebyshev interpolation, and examine the residual as above. Each discrete HOPM iteration costs $O\left(n^{3}\right)$ operations, which can be significantly higher than a continuous HOPM iteration, costing $O\left(r^{3}+\right.$ $r n \log n)$; recall the discussion in Section 2.1. Furthermore, for the discrete HOPM, the appropriate value of $n$ is not known a priori-observe that in Figure 6.1 (right), $n=10$ is insufficient to obtain full accuracy.

Sometimes the power method converges not to the largest singular quadruplet but to another one. Experiments suggest this happens with nonnegligible probability (see Section 6.2), although most of the time, the power method finds the largest singular quadruplet. For the purpose of finding the best rank-1 approximant, a simple remedy is to run the power method several times with multiple initial guesses and adopt the one with the largest singular value. Alternatively, one can use the first term of the multivariate adaptive cross approximation (MACA), in which one finds a low-rank approximation via the slices and fibers passing through a pivot value [2] or HOSVD of $f$ as the initial guess instead of using random ones.

singular values grows rapidly with both the dimension and size, and that the singular vector tuples form a positive-dimensional variety. Extending such results to the continuous case is an interesting open problem. 
6.1.1 Low-rank approximation via CP vs. greedy, general case

It is known that the greedy algorithm of approximating a tensor by successively finding and subtracting the best rank-1 approximant generally does not give the $\mathrm{CP}$ decomposition [28]. Indeed, the result in [62] can be seen to carry over to functions, to show that generic trivariate functions do not have a Schmidt-Eckart-Young decomposition (such functions need to have a weak two-orthogonal decomposition [62, Thm. 3.3]). We illustrate this in Figure 6.2, where the residual $\|f-\hat{f}\|$ is shown for an approximant $\hat{f}$ of varying rank, obtained by either the greedy approach or truncating the CP decomposition.
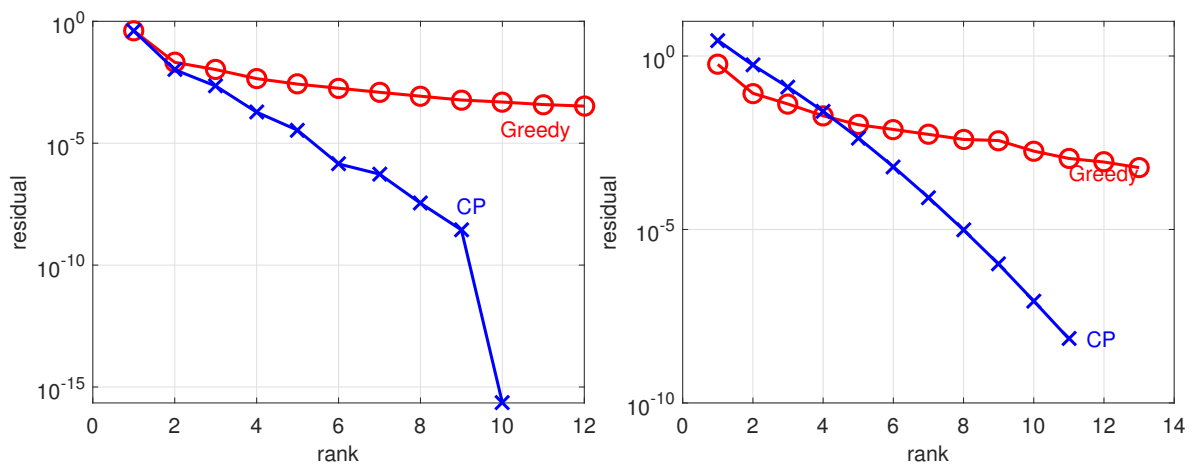

Fig. 6.2 Convergence of residual $\|f-\hat{f}\|$ via greedy rank-1 approximation and CP for non-odeco (in any direction) functions. Left: random rank-10 function with $\alpha_{j}=10^{-j}$. Right: $f(x, y, z)=\exp (x y z)$.

We verify as expected that the greedy approach does not give the optimal rank- $r$ approximants, except when $r=1$. With a rank-one or low-rank approximation, on the other hand, the greedy approach usually gives a smaller residual; this reflects the fact that it is indeed the best rank-1 approximant, which is not necessarily true for $\mathrm{CP}$ (unless the function is two-way odeco).

Note that the $\mathrm{CP}$ residual does not always go down to working precision $\approx 10^{-16}$; for example, for $f(x, y, z)=\exp (x y z)$ and the computed CP decomposition has residual $\approx 10^{-8}$, and this does not improve by changing the SVD tolerance in Algorithm 4; we observed similar behaviors for many functions. This seems to be caused by conditioning of the eigenvalue problem, in particular, the quasimatrix of eigenvectors (step 5 of Algorithm 4). For this reason we do not always recommend finding a compressed representation of a trivariate function by the $\mathrm{CP}$ decomposition.

\subsubsection{Low-rank approximation via CP vs. greedy, two-way odeco case}

For two-way odeco functions, we have shown in Proposition 5.1 that the leading $r$ $\mathrm{CP}$ factors give the best rank- $r$ approximation. A corollary of this result is that a greedy approach of successively subtracting the largest CP quadruplet (equal to the largest singular quadruplet) gives the $\mathrm{CP}$ decomposition and hence the best rank- $r$ approximation. We illustrate this in Figure 6.3. Here we generate a two-way odeco 
function as in (2.9), where we take $T$ to be a diagonal tensor of diagonal elements $10^{-j}, j=1,2, \ldots, 10$ and $B:=P Q_{B}$ and $C:=P Q_{C}$ where $P$ is an orthonormal quasimatrix of Legendre polynomials and $Q_{B}$ and $Q_{C}$ are $10 \times 10$ random orthonormal matrices. The equivalence between the $\mathrm{CP}$ and greedy curves (up to roundoff errors) illustrates the fact that for odeco or two-way odeco functions, the greedy approach and truncated CP both give the best rank- $r$ approximant for every $r$.
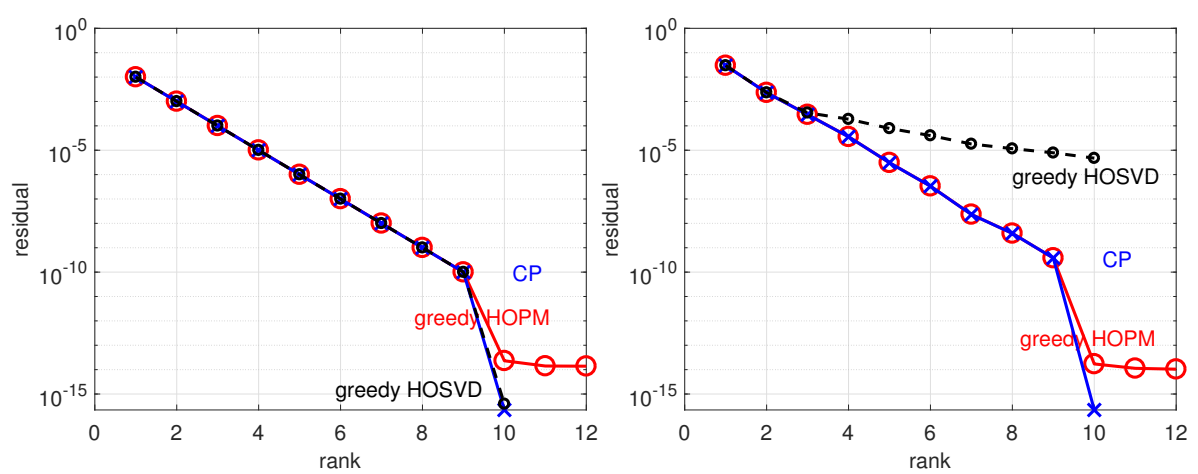

Fig. 6.3 For three-way odeco (left) and two-way odeco (right) functions, the SVD and CP are the same, so a greedy HOPM gives CP (up to floating-point errors). A greedy HOSVD, by contrast, gives CP for three-way odeco functions but not for two-way odeco.

\subsubsection{A nonstandard greedy approach}

It is known that for a trivariate function, HOSVD gives an approximation of trilinear rank $\left(r_{1}, r_{2}, r_{3}\right)$ that is quasi-optimal, within a factor of $\sqrt{3}$ (or more generally the square root of the dimension, cf. [23, Thm. 10.3]). As mentioned in the last two subsections, it is standard and natural to use HOPM (rather than HOSVD) at every step of the greedy approach. Nevertheless, here we examine another variant of greedy approach in which HOPM is replaced with HOSVD truncation of rank $(1,1,1)$ at every step. We observed that for three-way odeco functions, greedy HOSVD and greedy HOPM are equal and both optimal. In contrast, for two-way odeco functions greedy HOPM is optimal (first two parts of Proposition 5.1), but greedy HOSVD is not. We illustrate these in Figure 6.3.

\subsubsection{Finding a rank-one function in a subspace}

As a natural continuous analogue of the problem considered in [41], consider the following problem. Let $f, g, h$ be rank-one trivariate functions, and let $\hat{f}=f+c_{1} g+$ $c_{2} h$, which is of rank three. Given $\hat{f}, g, h$, we would like to find (or "recover") the function $f$ such that

1. $f$ is rank one, and

2. $\hat{f}, g, h$ and $f, g, h$ span the same subspace. 
Put another way, we are looking for a basis consisting of rank-one functions for the subspace spanned by $\hat{f}, g$, and $h$. In the discrete case, this problem has applications in data compression and image separation.

As also described in [40] a simple algorithm executes alternating projection between rank-one functions and the subspace of trivariate functions spanned by $\hat{f}, g$, and $h$. In that Example, projection to rank-one functions was done via the MACA process. Now equipped with a method for the best rank-one approximation, we replace MACA with HOPM; Figure 6.4 shows the results, which plot the error $\hat{f}_{\text {it }}-f$ in the $x$-mode after one through seven iterations.

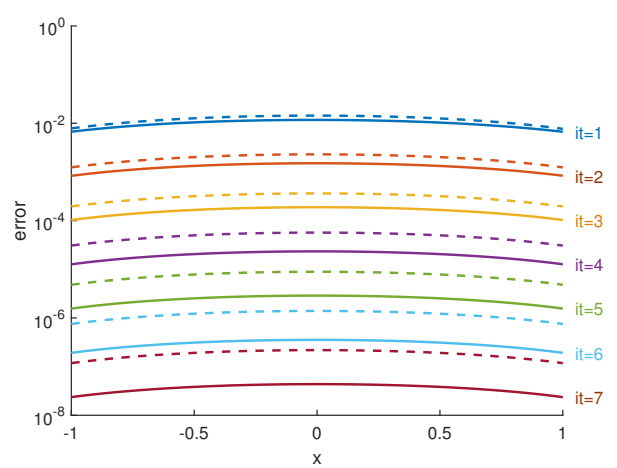

Fig. 6.4 Convergence of $x$-mode as iteration proceeds for finding a rank-one trivariate function in a subspace of trivariate functions. The dashed line shows the performance when the rank-1 extraction is done in an MACA manner.

We observe from the fact that the solid curves (HOPM) lie below the dashed ones (MACA), that by finding the correct best rank-1 approximant in each step, we improve the convergence.

\subsection{Computing more than one singular quadruplet with HOPM}

The HOPM usually converges to the dominant singular quadruplet. However, convergence to a different singular quadruplet is also possible. For example for the function ${ }^{5}$ $f(x, y, z)=\left|((x+i y) \exp (i z))^{2}-1\right|^{2}$ defined over $[-1.5,1.5] \times[-1.5,1.5] \times[-6,6]$ we have computed the singular values $\sigma_{1}=55.74$ and $\sigma_{2}=22.46$. The corresponding singular functions in each mode are depicted in Figure 6.5. In this example HOPM converged to the largest singular quadruplet with $\sigma_{1}=55.74$ about $80 \%$ of the random runs, the remaining runs all converging to $\sigma_{2}=22.46$. In most other examples that we tried, HOPM converged to the largest singular quadruplet with (often much) higher probability.

\subsection{Eigenvalues of symmetric functions}

As mentioned in Section 3.1, if the shift parameter of the SS-HOPM is chosen appropriately, then the eigenvalues and eigenvectors of symmetric discrete tensors might

\footnotetext{
5 This is the function doublehelix from the Chebfun gallery.
} 

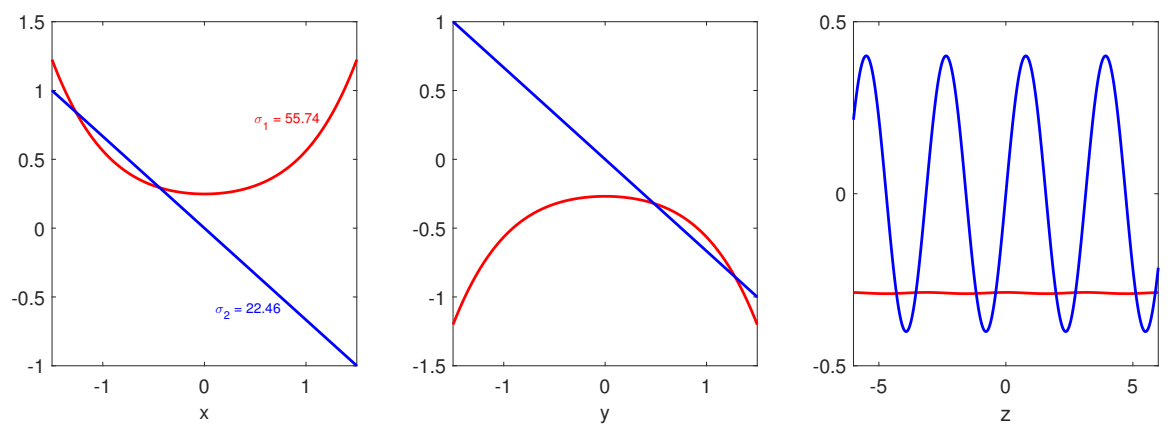

Fig. 6.5 Two leading singular functions of the doublehelix function, in the $x, y, z$ modes.

be computed in a way that the underlying optimization problem reduces to a convex problem [29]. For symmetric functions, the infinite-dimensional counterpart, we observe similar phenomena. Figure 6.7 shows a typical convergence history, which is again linear with the eigenvalues converging like the square of the residual.

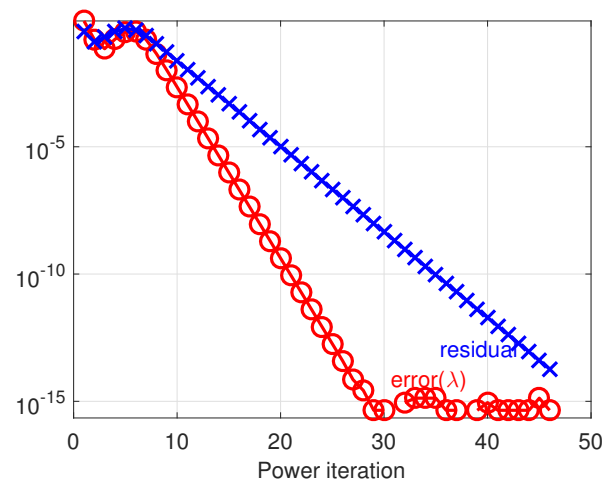

Fig. 6.6 Typical convergence of power method for an eigenpair of a symmetric function $f(x, y, z)=\sin (x+$ $y+z$ ) with shift 1 (shift 0 often results in nonconvergence, see Figure 6.7). As for the singular values, the eigenvalue accuracy scales like the residual squared.

The shift also gives the flexibility to compute eigenpairs other than the largest one, unlike the counterpart for singular values presented above. We give an illustration in Figure 6.7, which shows the history of the Rayleigh quotient for different values of the shift parameter. Note that without a shift, the power method did not converge. It would be of interest to find the singular quadruplets that are not the largest by a similar shifting technique. 


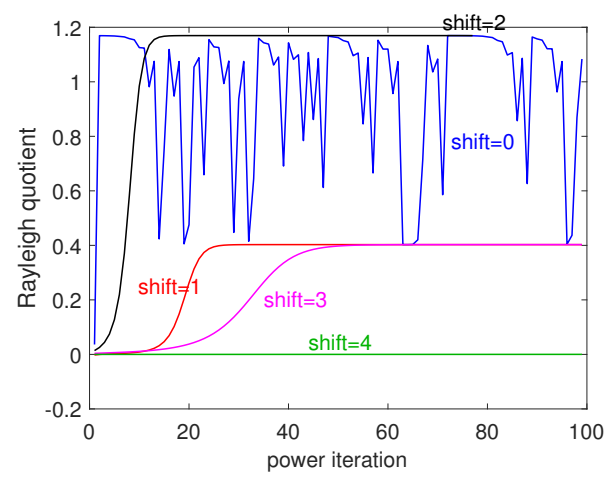

Fig. 6.7 Typical convergence of power method for an eigenpair of a symmetric function $f(x, y, z)=\sin (x+$ $y+z$ ) with varying shifts

\subsection{Decay of CP values}

We have proved in Section 5 that for two-way odeco functions, the CP values decay exponentially, which represent the quality of the best rank- $r$ approximation, and that singular values and $\mathrm{CP}$ values are equivalent for three-way odeco functions.

One difficulty to illustrate the geometric decay of $\mathrm{CP}$ values is that it is nontrivial to generate a two-way odeco function without specifying the $\mathrm{CP}$ values. For example, $f(x, y, z)$ as in (2.9) with $T$ being the diagonal tensor with $1 \mathrm{~s}$ on the diagonal, and $B$ and $C$ orthonormal, is a $(y, z)$ two-way odeco function, but its $C P$ values are specified explicitly by construction: they are the 2-norms of the columns of the quasimatrix $A$. Another example of a two-way odeco function is $f(x, y, z):=G(x, y) w(z)$, which involves only one univariate factor in one of the variables. Then the existence of SVD for the bivariate function $G(x, y)$ guarantees that $f$ is two-way odeco. In this case the $\mathrm{CP}$ values of $f$ are the singular values of $G$ multiplied by the 2-norm of $w$.

Meanwhile, we do not know whether the CP values would decay similarly for general functions. Nonetheless, experiments suggest that CP-values decay exponentially for general smooth functions, even if they are not two-way odeco, as illustrated in Figure 6.8. Making this precise is left as an open problem.

Acknowledgements The authors would like to thank Alex Townsend, André Uschmajew and Nick Vannieuwenhoven for helpful comments on the manuscript. We thank the referees for their comments and constructive suggestions.

The work of the first author was in part supported by a grant from IPM (No. 96150051). The second author was supported by JSPS as an Overseas Research Fellow.

\section{References}

1. Atkinson, K., Han, W.: Theoretical Numerical Analysis. Springer (2005)

2. Bebendorf, M.: Adaptive cross approximation of multivariate functions. Constr. Approx. 34(2), 149179 (2011)

3. Benson, A.R., Gleich, D.F., Lim, L.H.: The spacey random walk: A stochastic process for higher-order data. SIAM Rev. 59(2), 321-345 (2017) 


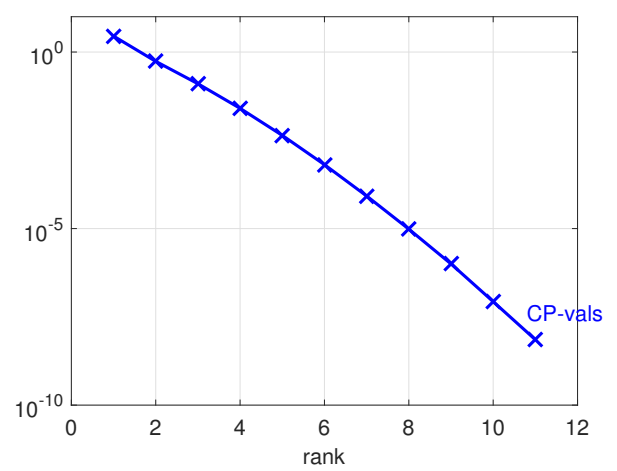

Fig. 6.8 CP values of analytic (entire) function $\exp (x y z)$.

4. Beylkin, G., Mohlenkamp, M.J.: Numerical operator calculus in higher dimensions. Proc. Natl. Acad. Sci. 99(16), 10,246-10,251 (2002)

5. Beylkin, G., Mohlenkamp, M.J.: Algorithms for numerical analysis in high dimensions. SIAM J. Sci. Comp 26(6), 2133-2159 (2005)

6. Blekherman, G., Teitler, Z.: On maximum, typical and generic ranks. Mathematische Annalen 362(34), 1021-1031 (2015)

7. Bigoni, D., Engsig-Karup, A.P., Marzouk, Y. M.: Spectral tensor-train decomposition. SIAM Journal on Scientific Computing 38(4), A2405- A2439 (2016)

8. Chevreuil, M., Lebrun, R., Nouy, A., Rai, P.: A least-squares method for sparse low rank approximation of multivariate functions. SIAM/ASA J. Uncertainty Quantification 3(1), 897-921 (2015)

9. Courant, R., Hilbert, D.: Methods of Mathematical Physics, vol. 1. CUP Archive (1965)

10. De Lathauwer, L.: A link between the canonical decomposition in multilinear algebra and simultaneous matrix diagonalization. SIAM J. Matrix Anal. Appl. 28(3), 642-666 (electronic) (2006).

11. De Lathauwer, L., Comon, P., De Moor, B., Vandewalle, J.: Higher-order power method. Nonlinear Theory and its Applications, NOLTA95 1 (1995)

12. De Lathauwer, L., De Moor, B., Vandewalle, J.: A multilinear singular value decomposition. SIAM J. Matrix Anal. Appl. 21, 1253-1278 (2000)

13. De Lathauwer, L., De Moor, B., Vandewalle, J.: Computation of the canonical decomposition by means of a simultaneous generalized Schur decomposition. SIAM J. Matrix Anal. Appl. 26(2), 295327 (electronic) (2004).

14. Demmel, J.: Applied Numerical Linear Algebra. SIAM, Philadelphia, USA (1997)

15. De Silva, V., Lim L-H.: Tensor rank and the ill-posedness of the best low-rank approximation problem. SIAM J. Matrix Anal. Appl. 30(3), 1084-1127 (2008)

16. Domanov, I., Lathauwer, L.D.: Canonical polyadic decomposition of third-order tensors: Reduction to generalized eigenvalue decomposition. SIAM J. Matrix Anal. Appl. 35(2), 636-660 (2014)

17. Driscoll, T.A., Hale, N., Trefethen, L.N.: Chebfun Guide. Pafnuty Publications, Oxford (2014)

18. Engel, E., Dreizler, R.M.: Density Functional Theory: An Advanced Course. Springer (2011)

19. Falcó, A., Hackbusch, W.: On minimal subspaces in tensor representations. Found. Comput. Math. 12(6), 765-803 (2012)

20. Golub, G.H., Van Loan, C.F.: Matrix Computations. Johns Hopkins University Press (2013)

21. Gorodetsky, A.A., Karaman, S., Marzouk, Y.M.: Function-train: A continuous analogue of the tensortrain decomposition. arXiv preprint arXiv:1510.09088v2 (2016)

22. van Groesen, E.: Applied Analytical Method. Lecture notes for Applied Analysis \& Mathematical Physics (March 2001)

23. Hackbusch, W.: Tensor Spaces and Numerical Tensor Calculus, Springer Series in Computational Mathematics, vol. 42. Springer, Heidelberg (2012).

24. Hashemi, B., Trefethen, L.N.: Chebfun in three dimensions. SIAM J. on Sci. Comp., 39(5), C341-363 (2017)

25. Hille, E., Tamarkin, J.D.: On the characteristic values of linear integral equations. Acta Math. 57(1), $1-76$ (1931). 
26. Kofidis, E., Regalia, P.A.: On the best rank-1 approximation of higher-order supersymmetric tensors. SIAM J. Matrix Anal. Appl. 23(3), 863-884 (2002)

27. Kolda, T.: Orthogonal tensor decompositions. SIAM J. Matrix Anal. Appl. 23(1), 243-255 (2001)

28. Kolda, T.G., Bader, B.W.: Tensor decompositions and applications. SIAM Rev. 51(3), 455-500 (2009)

29. Kolda, T.G., Mayo, J.R.: Shifted power method for computing tensor eigenpairs. SIAM J. Matrix Anal. Appl. 32(4), 1095-1124 (2011)

30. Kress, R.: Linear Integral Equations. Springer (2014)

31. Kruskal, J.B.: Three-way arrays: rank and uniqueness of trilinear decompositions, with application to arithmetic complexity and statistics. Linear Algebra Appl. 18(2), 95-138 (1977)

32. Kruskal, J.B.: Rank, decomposition, and uniqueness for 3-way and n-way arrays. In: Multiway Data Analysis, R. Coppi and S. Bolasco, eds., North-Holland, p. 718 (1989)

33. Leurgans, S.E., Ross, R.T., Abel, R.B.: A decomposition for three-way arrays. SIAM J. Matrix Anal. Appl. 14(4), 1064-1083 (1993).

34. Li, W., Ng, M.K.: On the limiting probability distribution of a transition probability tensor. Linear Multilinear A. 62(3), 362-385 (2014)

35. Lickteig, T.: Typical tensorial rank. Linear Algebra Appl. 69, 95-120 (1985)

36. Lim, L.H.: Singular values and eigenvalues of tensors: a variational approach. In: Proceedings of the IEEE International Workshop on Computational Advances in Multi-Sensor Adaptive Processing (CAMSAP '05), pp. 129-132 (2005)

37. Little, G., Reade, J.: Eigenvalues of analytic kernels. SIAM J. Math. Anal. 15, 133-136 (1984)

38. Mercer, J.: Functions of positive and negative type and their connection with the theory of integral equations. Phil. Trans. R. Soc. Lond. A 209, 415-446 (1909)

39. Moler, Cleve B. and Stewart, G. W.: An algorithm for generalized matrix eigenvalue problems. SIAM J. Numer. Anal. 10(2), 241-256 (1973)

40. Nakatsukasa, Y.: Finding a trivariate basis of rank-one functions. Chebfun Example. June 2016, http://www. chebfun.org/examples/approx3/FindingRankOne.html

41. Nakatsukasa, Y., Soma, T., Uschmajew, A.: Finding a low-rank basis in a matrix subspace. Math. Prog. 162(1-2), 325-361 (2017)

42. Qi, L.: Eigenvalues of a real supersymmetric tensor. J. Symb. Comput. 40(6), 1302-1324 (2005)

43. Oseledets, I.V.: Constructive representation of functions in low-rank tensor formats. Constructive Approximation 37(1), 1-18 (2013)

44. Regalia, P.A., Kofidis, E.: The higher-order power method revisited: convergence proofs and effective initialization. In: Acoustics, Speech, and Signal Processing, 2000. ICASSP'00. Proceedings. 2000 IEEE International Conference on, vol. 5, pp. 2709-2712. IEEE (2000)

45. Robeva, E., Seigal, A.: Singular vectors of orthogonally decomposable tensors. Linear Multilinear A. pp. 1-15 (2017)

46. Sanchez, E., Kowalski, B.R.: Tensorial resolution: a direct trilinear decomposition. Journal of Chemometrics 4(1), 29-45 (1990)

47. Schmidt, E.: Zur Theorie der linearen und nichtlinearen Integralgleichungen. I Teil. Entwicklung willkuerlichen Funktionen nach System vorgeschriebener. Math. Ann. 63, 433-476 (1907)

48. Schultz, T., Seidel, H.P.: Estimating crossing fibers: A tensor decomposition approach. IEEE Trans. Vis. Comput. Graph. 14(6) (2008)

49. Spatschek, R., Eidel, B.: Driving forces for interface kinetics and phase field models. Int. J. Solids Struct. 50(14), 2424-2436 (2013)

50. Stakgold, I., Holst, M.J.: Green's Functions and Boundary Value Problems. John Wiley \& Sons (2011)

51. Stewart, G.W.: On the early history of the singular value decomposition. SIAM Rev. 35(4), 551-566 (1993)

52. Stewart, G.W.: Afternotes Goes to Graduate School: Lectures on Advanced Numerical Analysis. SIAM (1998)

53. Strassen, V.: Rank and optimal computation of generic tensors. Linear Algebra and its Applications 52, 645-685 (1983)

54. Sturmfels, B.: Tensors and their eigenvectors. Notices Amer. Math. Soc. 63(6) (2016)

55. Townsend, A.: Computing with functions in two dimensions. Ph.D. thesis, University of Oxford (2014)

56. Townsend, A., Trefethen, L.N.: An extension of Chebfun to two dimensions. SIAM J. Sci. Comp 35(6), C495-C518 (2013)

57. Townsend, A., Trefethen, L.N.: Continuous analogues of matrix factorizations. Proc. Roy. Soc. A 471(2173), 20140,585 (2015) 
58. Trefethen, L.N.: Householder triangularization of a quasimatrix. IMA J. Numer. Anal. 30(4), 887-897 (2010)

59. Trefethen, L.N.: Approximation Theory and Approximation Practice. SIAM (2013)

60. Uschmajew, A.: Regularity of tensor product approximations to square integrable functions. Constr Approx. 34, 371-391 (2011)

61. Uschmajew, A.: A new convergence proof for the higher-order power method and generalizations. Pac. J. Optim. 11, 309-321 (2015)

62. Vannieuwenhoven, N., Nicaise, J., Vandebril, R., Meerbergen, K.: On generic nonexistence of the Schmidt-Eckart-Young decomposition for complex tensors. SIAM J. Matrix Anal. Appl. 35(3), 886903 (2014)

63. Zhang, T., Golub, G.H.: Rank-one approximation to high order tensors. SIAM J. Matrix Anal. Appl. 23(2), 534-550 (2001) 